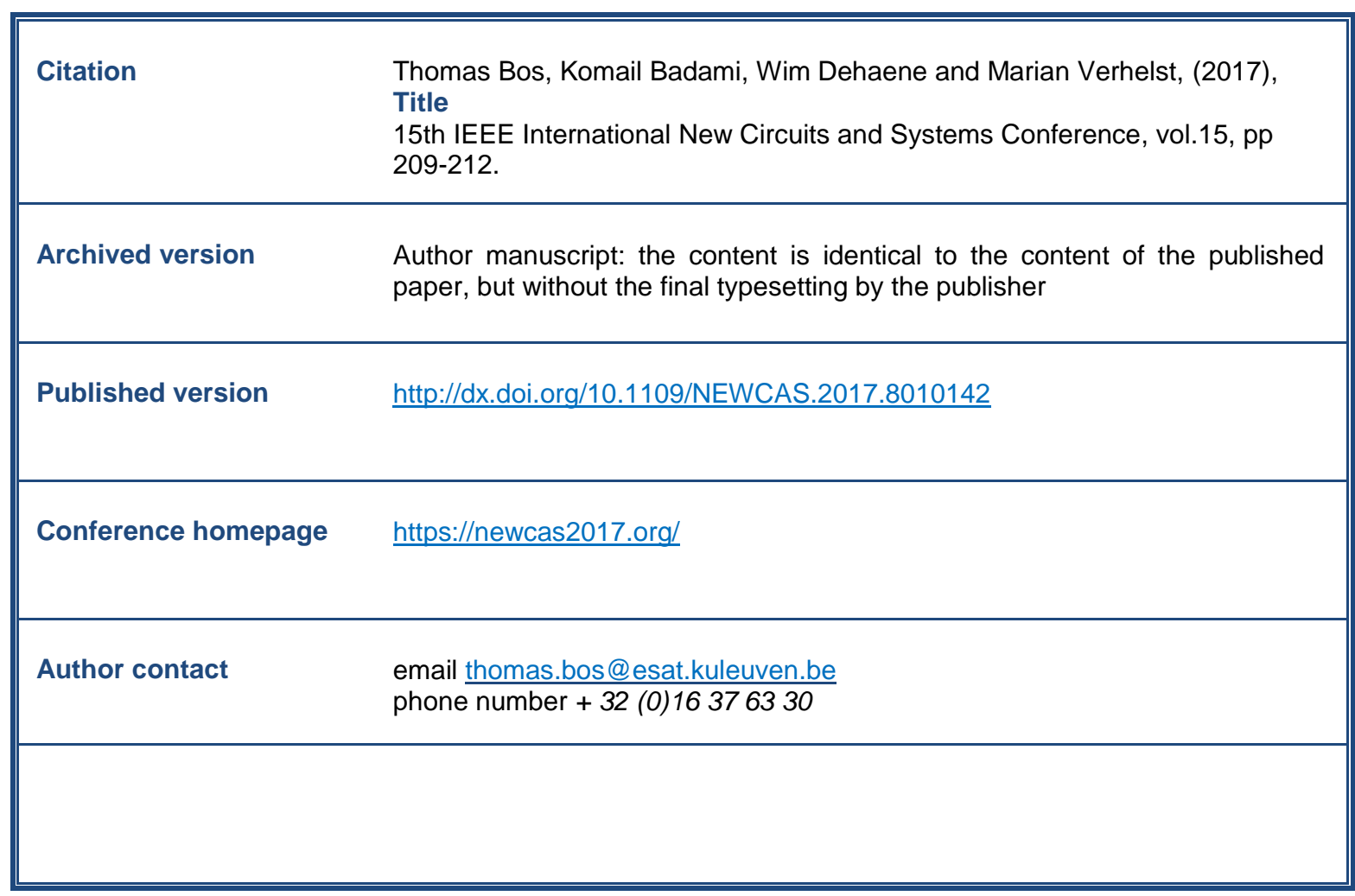

(article begins on next page) 


\title{
An 8-11b 320kS/s Resolution Scalable Noise Shaping SAR ADC
}

\author{
Thomas Bos ${ }^{1}$, Komail Badami, Wim Dehaene and Marian Verhelst \\ KU Leuven - ESAT - MICAS, Leuven, Belgium \\ ${ }^{1}$ thomas.bos@esat.kuleuven.be
}

\begin{abstract}
This work presents an 8 - 11 b resolution scalable and energy efficient ADC, using the oversampled and noise shaping SAR architecture in 90nm UMC CMOS process. Further, the proposed ADC simplifies the design of the noise shaping filter to enable the use of a first order switched capacitor low pass filter for shaping the comparator noise and the in band quantization noise. The ADC design alleviates the matching concerns by using only an $8 \mathrm{~b}$ capacitive DAC, and allows to configure the ADC architecture from an 8b traditional SAR ADC up to an 11b ADC by enabling the oversampling and noise shaping loops within the SAR architecture. This ADC is designed to operate with 8-11b resolution, up to $320 \mathrm{kS} / \mathrm{s}$, achieving a power scaling from $80 \mathrm{nW}$ to $1.5 \mu \mathrm{W}$, resulting in an average FoM of 30fJ/conv-step.
\end{abstract}

\section{INTRODUCTION}

Low power ADCs in sensor front ends often target resolution versus power scalability, to minimize the system power consumption as a function of the complexity of the current computation task [1]. Typically, such ADCs operate in lowmedium frequency range with resolution ranging between 8 to $12 \mathrm{~b}$ depending on the application. The charge redistribution SAR ADC provides very high energy efficiency and is well suited for implementation in advanced CMOS technologies [2]. Yet, its operation is often limited to less than $10 \mathrm{~b}$ due to the increased power consumption of the noise limited comparator [2] and the matching requirements of the DAC capacitor array. Oversampled ADC architectures alleviate this ENOB limit by trading-off bandwidth, easily available in scaled CMOS technologies, for higher resolutions. Such oversampled architectures allow further resolution enhancement by adding a noise shaping feedback loop, which shapes the comparator and the in-band quantization noise to higher frequencies, thus enhancing the in-band SNR [3].

This work introduces an 8 to $11 \mathrm{~b}$ resolution scalable ADC by combining the architecture of the oversampled, noise shaping converter with an $8 \mathrm{~b}$ capacitive DAC based, energy efficient SAR ADC. Such an architecture was first used in [4] for a fixed resolution, and is here improved along the following lines. First, it enables a configurable ADC architecture that allows to scale resolution from $8 \mathrm{~b}$ with a traditional SAR $\mathrm{ADC}$, to up to $11 \mathrm{~b}$ by selectively enabling the oversampling and the noise shaping loops in the SAR architecture. Secondly, it simplifies the noise shaping filter design down to a simple, first order switched capacitor based filter. The ADC design presented in this paper targets speech processing applications with a sampling rate of up to $320 \mathrm{kS} / \mathrm{s}$ for a signal bandwidth of up to $10 \mathrm{kHz}$.

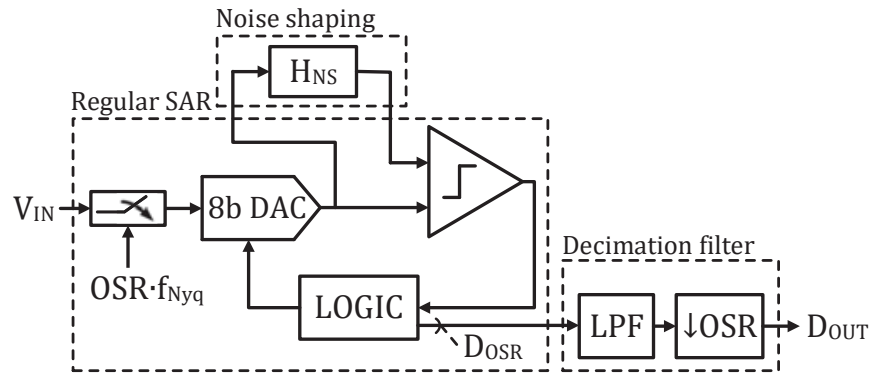

Fig. 1. System level diagram for the resolution scalable, oversampled noise shaping SAR ADC.

The paper is organised as follows: Section II presents the system architecture, describes the mathematical model for the resolution scalable, oversampled noise shaped SAR ADC and provides simulation results from a MATLAB model. Next, Section III details the circuit design for different blocks in the oversampled and noise shaping SAR ADC. Section IV details the particulars of the circuit simulation setup and provides the ADC simulation results in 90nm UMC CMOS process.

\section{Resolution Scalable Oversampled Noise SHAPING SAR ARCHITECTURE}

The system diagram for the proposed resolution scalable, oversampled noise shaping SAR is depicted in figure 1. The crucial blocks are: (a) a regular SAR structure consisting of the DAC, comparator and logic; (b) a noise shaping loop; and (c) a decimation filter. The input voltage $V_{I N}$ is sampled at $f_{s}=O S R \cdot f_{N y q}$ on the capacitive DAC. Based on the comparator decision, the digital logic runs the classical binary search algorithm to find the nearest digital output code. This ADC architecture enables resolution scaling as follows. In the lowest resolution mode, the noise shaping loop and the decimation filter are disabled and the ADC operates as a regular 8b SAR ADC with $f_{s}=f_{N y q}=2 \cdot B W_{i n}$. For higher resolution, the noise shaping loop is enabled and by selecting OSR from 1 to 16 , the ADC resolution can be varied from 8 to $11 \mathrm{~b}$.

In a regular SAR ADC, the residual charge on the DAC is discarded at the end of conversion cycle. However, in the oversampled noise shaping SAR ADC, the residual charge Q is coupled back to the input of the SAR comparator, while the LSB decision is also fed back to the DAC. In such an architecture it can be proven that [4]: 

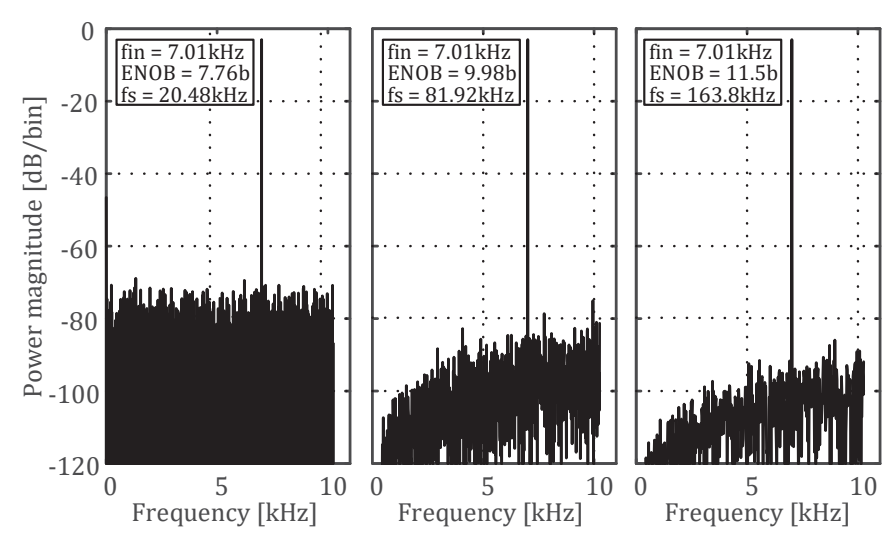

Fig. 2. MATLAB simulation results with a $7.01 \mathrm{kHz}, 2 \mathrm{Vppd}$ input signal, depicting resolution scalability with oversampling ratio. Left: the ideal regular 8 bit SAR. Center: $4 x$ oversampling with first order noise shaping. Right: $8 x$ oversampling with first order noise shaping. Ideal decimation is used in the MATLAB simulations.

$$
\begin{gathered}
V_{D A C}=D_{O U T}-V_{I N} \triangleq V_{Q} \\
D_{O S R}(z)=V_{I N}(z)+\frac{1}{1+H_{N S}(z)} \cdot V_{Q}(z)
\end{gathered}
$$

where $H_{N S}$ is the transfer function used for noise shaping and $V_{Q}$ is the quantization residue. Intuitively, analog to digital conversion with Eq. 2 is very similar to that of a delta-sigma converter. The input signal $V_{I N}$ has a unity gain transfer function to the digital output $D_{O S R}$, whereas the quantization error $V_{Q}$ is pushed out of band towards higher frequencies. The decimation filter at the output suppresses the high frequency content dominated by the quantization noise. Hereby, the desired signal at low frequency is unaffected and a higher ENOB is achieved than the fixed resolution of the DAC. To push the quantization noise to higher frequency, the noise shaping filter $H_{N S}$ should be low pass. Our approach uses a simple, switched capacitor based low pass filter with a transfer function as shown below:

$$
H_{N S}(z)=\frac{z^{-1}}{1-z^{-1}}
$$

This filter is chosen, over the filter of [4], because it provides a more constant increment in ENOB, which is useful for resolution scalability, whereas the filter of [4] is optimized towards a fixed OSR of 4. Further, this filter also consumes less power because it allows to use smaller capacitors.

The complete architecture in figure 1 is modeled in a MATLAB environment to check the above theoretical assertions. Figure 2-left shows the output magnitude spectrum of an $8 \mathrm{~b}$ fully differential SAR ADC with a $7.01 \mathrm{kHz}$ sinusoidal input signal with an amplitude $2 \mathrm{Vppd}$, sampled at $20.48 \mathrm{kHz}$. When the noise shaping loop and ideal decimation filter are enabled, it can be seen that the resolution increases from a DAC limited value of $8 \mathrm{~b}$ to $9.9 \mathrm{~b}$ with an OSR of 4 (Fig. 2-center) and to $11.5 \mathrm{~b}$ for an OSR of 8 (Fig. 2-right).
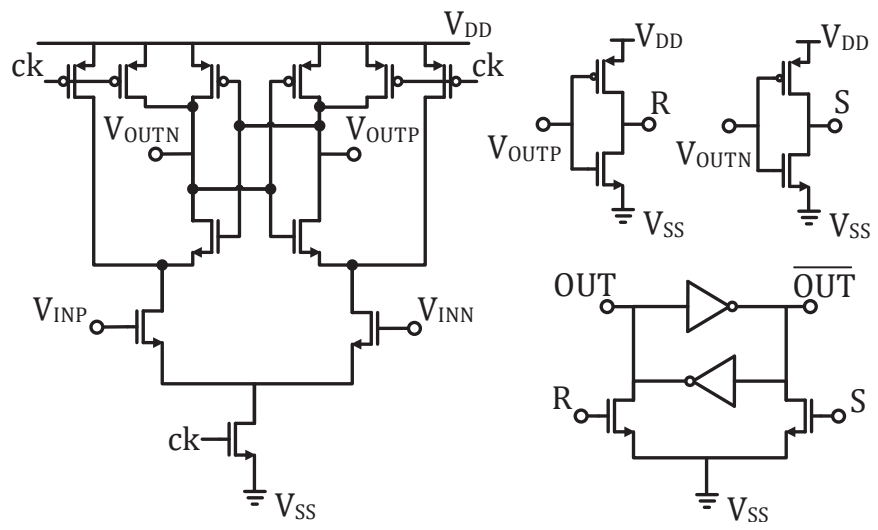

Fig. 3. Left: Circuit details of the strong arm latch based comparator. Right: the invertors to assure equal loading on the comparator output pins, and the active high RS-latch to assure reliable interfacing to the logic.

\section{CirCuit Design OF THE OVERSAMPLED Noise SHAPING SAR ADC}

This section details the circuit design of the resolution scalable, oversampled noise shaping SAR in 90nm UMC CMOS process. All following circuits are presented in single ended graphs, which in reality are designed fully differential. Subsection A details the blocks of the regular SAR ADC, Subsection B explains the implementation of the oversampling blocks, while Subsection C extends the design to include the noise shaping loop.

\section{A. Inner $S A R A D C$ design}

A regular SAR ADC has the $\mathrm{DAC}$, the comparator and the control logic as crucial functional blocks. This ADC uses a fully differential $8 \mathrm{~b}$ binary weighted capacitive DAC. The unit capacitance $C_{u}$ for this DAC is chosen to be a $5 \mathrm{fF}$ MOM capacitor. The input signal is bottom plate sampled on the capacitor array, while the top plate samples the common mode $V_{C M}$. The thermal noise of the single ended DAC estimated to be $56.9 \mu \mathrm{Vrms}$ is a factor of about 9x smaller than the quantization noise with $11 \mathrm{~b}$ accuracy.

A strong arm latch is used as the comparator and is designed for only $8 \mathrm{~b}$ performance, as a very important advantage of the oversampled and noise shaped SAR ADC is that the comparator noise is also shaped along with the quantization noise to out of band frequencies. The architecture of the comparator is shown in figure 3 , along with its interface to the digital logic. The strong arm latch is chosen, because of its speed and power advantages over a static comparator [5]. Furthermore, in order to enable scalable energy efficiency in function of the configurable ADC resolution, all the blocks of the regular SAR ADC are completely dynamic. Hence, power consumption scales linearly with the sampling frequency of the ADC, and hence with the desired resolution.

The digital SAR logic consists of a ring oscillator which generates the timing and control signals, and a code register for the control of the DAC, as used in regular SAR ADCs [6]. 


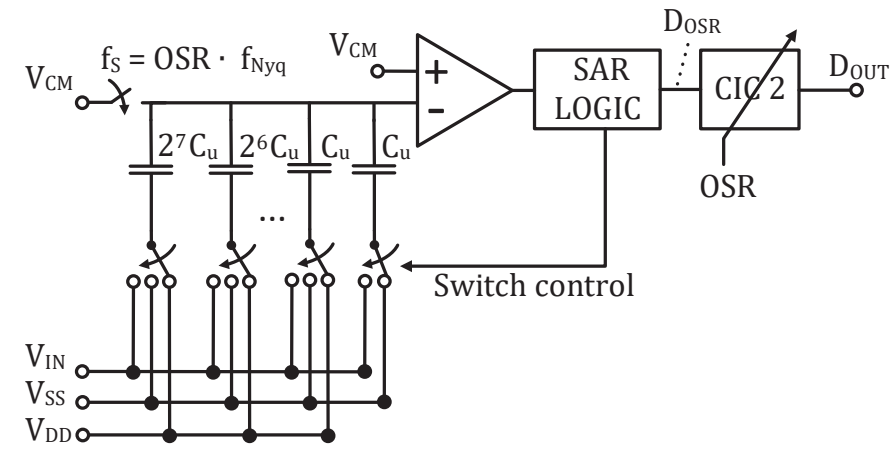

Fig. 4. Schematic depicting the signal flow through the oversampling blocks: the input sampling switches, the common mode DAC switch and the OSRscalable CIC filter that enables decimation of $D_{O S R}$ to $D_{O U T}$.

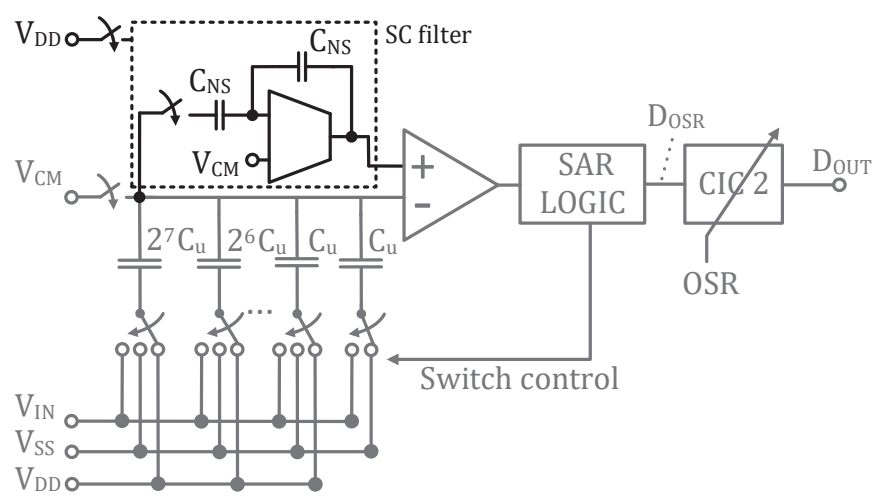

Fig. 5. Schematic illustrating the integration of the noise shaping loop (black) on the oversampling signal path (gray): the first order SC integrator samples and integrates the DAC voltage at the end of each SAR conversion. This SC filter is powered down at an OSR of 1 .

\section{B. Oversampling components design}

Figure 4 depicts the signal path for the oversampling operation in a regular SAR ADC. The sampling switch uses a transmission gate architecture, and is sized to achieve linearity of $14.5 \mathrm{~b}$. The top plate of the DAC samples the common mode voltage $V_{C M}$ using a bootstrapped switch to reduce the parasitic capacitance on the top plate, while meeting the necessary linearity specification. The oversampled SAR output $D_{O S R}$, is fed to the OSR-scalable CIC filter.

Hardware and power efficient second order CIC filters are used for the low pass filtering and decimation of the oversampled digital signal $D_{O S R}$. Also here, this filter type is chosen because of its power scalability with resolution, which is affected by scaling of the sampling frequency. The main drawback of the CIC filter is the high attenuation in the passband around Nyquist frequency.

\section{Noise shaping components design}

Figure 5 depicts the signal path for the noise shaping loop along with the oversampling path. The DAC voltage is sampled on the noise shaping capacitor $C_{N S}$ at the end of the SAR conversion cycle, and integrated by a first order switched capacitor integrator. The output of the DAC at the

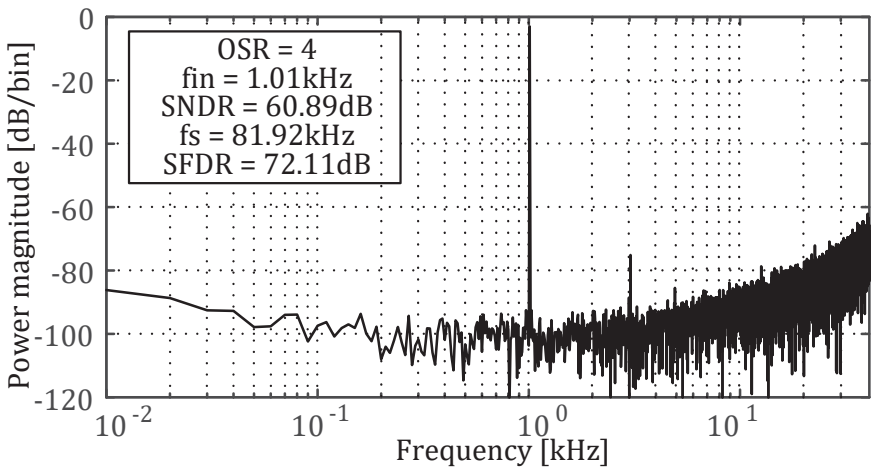

Fig. 6. The 8192 points based FFT spectrum of the oversampled digital output signal $D_{O S R}$ for a $2 \mathrm{Vppd}, 1.01 \mathrm{kHz}$ input signal. The ADC sampling frequency is $81.92 \mathrm{kHz}$, yielding an OSR of 4 .

end of the SAR conversion cycle is always between $V_{C M}$ and $V_{C M}-V_{L S B}$. This allows the use of a simple telescopic cascode OTA, as the signal swing at the input and output is relative small. The capacitor value $C_{N S}$ was chosen to be $50 \mathrm{fF}$ to reduce the noise contribution of the noise shaping loop. The noise shaping loop is disabled for the lowest resolution of $8 \mathrm{~b}$, as it allows to power down the OTA, to obtain minimal power consumption at all OSR values.

\section{Simulation Results}

This section details the setup and the simulation results for the oversampled and noise shaped SAR ADC, designed in $90 \mathrm{~nm}$ UMC CMOS. All circuits use a supply voltage of $1.2 \mathrm{~V}$. All displayed numbers and spectra are obtained with the Spectre simulator on transient simulations of $100 \mathrm{~ms}$. Transient noise is included in these simulations with $f_{\text {noise, } \max }=$ $100 \mathrm{MHz}$ and $f_{\text {noise, } \text { min }}=10 \mathrm{~Hz}$, where noise contribution from all the transistors in the analog signal path is included. Further, a transient simulation including a $1 \%$ relative mismatch on the DAC capacitor sizes resulted in only up to $0.1 \mathrm{~b}$ degradation in the ENOB. Finally, coherent sampling with 8192 points was used to estimate the FFT spectrum.

The magnitude spectrum for a differential input signal of $1.01 \mathrm{kHz}$ and $2 \mathrm{Vppd}$, and a sampling frequency of $81.92 \mathrm{kHz}$ for an OSR of 4, is shown in figure 6. Using an ideal low pass filter with a cut off frequency of $f_{N y q} / 2=10.24 \mathrm{kHz}$, yields an SNDR of $60.89 \mathrm{~dB}$ and corresponding ENOB of 9.82b.

Figure 7 shows the variation of ENOB versus input frequency for an OSR of 4. At higher input frequency, the ideal LPF curve slightly improves in ENOB, due to the large attenuation of the undesired harmonics which fall outside the passband of the decimation filter. The CIC2 filter achieves good ENOB at low to medium input frequencies. However, at the designed input bandwidth of $10.24 \mathrm{kHz}$, the large passband attenuation results in a degradation of about $1 \mathrm{~b}$ in ENOB.

Figure 8 (left Y-axis) illustrates the scaling of ENOB w.r.t. OSR. At an OSR of 1, the noise shaping filter is disabled, resulting in an ENOB of $7.8 \mathrm{~b}$ in the regular SAR mode. For higher resolutions, the design allows to increase the OSR up to 16 , and with noise shaping enabled, the resolution increases 


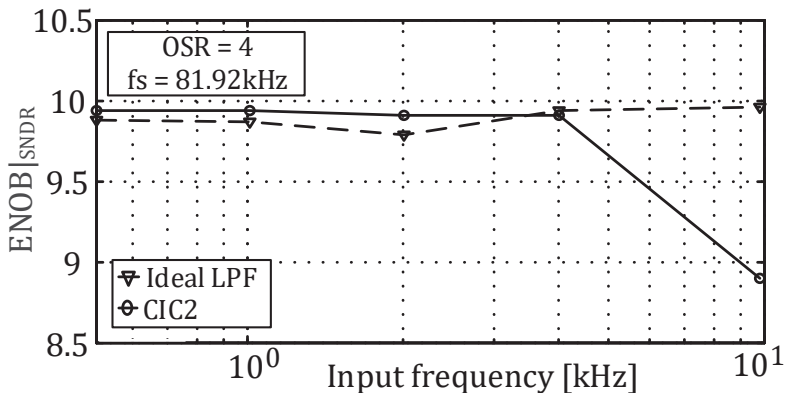

Fig. 7. ENOB versus input frequency for an OSR of 4. Decimation with an ideal (dotted) and CIC2 (solid) low pass filter with a cut off frequency of $10.24 \mathrm{kHz}$.

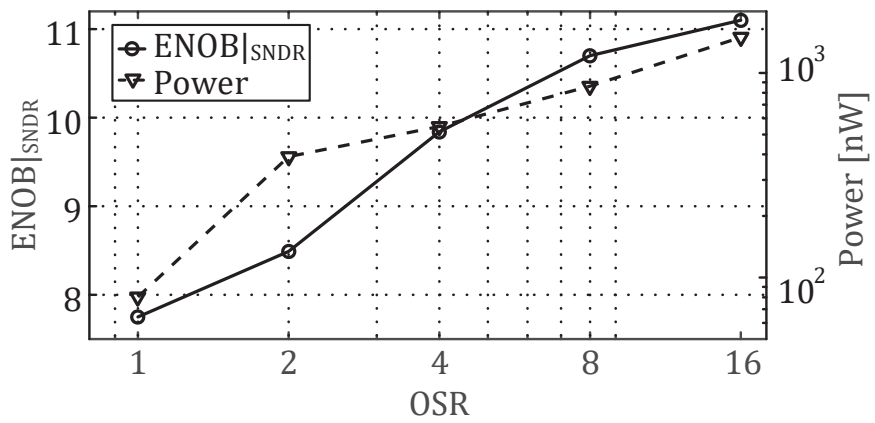

Fig. 8. ENOB (solid, left Y-axis) and Power (dashed, right Y-axis) versus OSR for an input frequency of $1 \mathrm{kHz}$.

up to an ENOB of $11 \mathrm{~b}$. Further, figure 8 (right Y-axis) also depicts the power consumption of the resolution scalable ADC as a function of the OSR. It can be seen that, by tuning the OSR, the ADC enables a power versus ENOB trade-off.

The power budget of the main blocks is represented in figure 9 for an OSR of 4 and 16. It can be seen that the power consumption of the DAC and the noise shaping loop is dominant at an OSR of 4. However, at OSR of 16, the power consumption is mainly dominated by the DAC due to the increase in sampling rate. The CIC filter is not included in the power estimation. The overall ADC power consumption sums up to $548 \mathrm{nW}$ at OSR of 4 and increases to $1.5 \mu \mathrm{W}$ at an OSR of 16. The FoM for the ADC is nearly constant and has an average value of $30 \mathrm{fJ} / \mathrm{conv}$-step due to a proportional increase in ENOB with an increase in power consumption.

Table I compares this design with the state of the art ADCs with low-medium sampling frequency and $10 \mathrm{~b}$ resolution. The FoM of the proposed ADC is comparable to the state of the art ADCs. However, Ref. [8] exhibits a significantly better FOM, mainly due to the use of a very small unit capacitance of $0.25 \mathrm{fF}$. Such a small unit capacitance was beyond the scope of this work, but can be combined with ideas presented here for further improvement in FoM.

\section{CONCLUSiON}

This work presents an $8-11 \mathrm{~b}$ resolution scalable, oversampled and noise shaped SAR ADC with a simplified noise shaping filter. Such an ADC architecture benefits from the

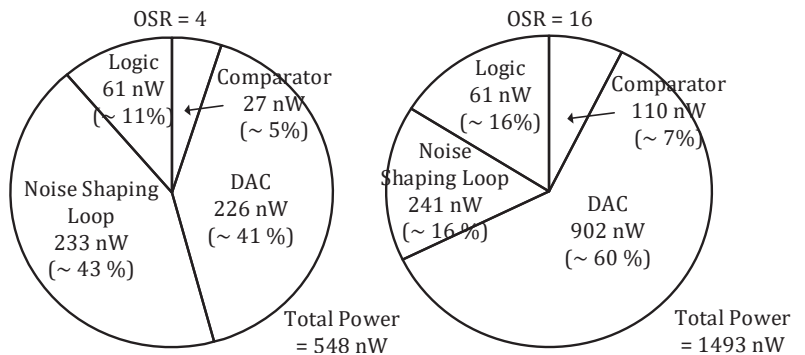

Fig. 9. Power breakdown of the resolution scalable ADC for OSR of 4 (left) and OSR of 16 (right).

TABLE I

COMPARISON TABLE

\begin{tabular}{|c|c|c|c|c|c|}
\hline & {$[7]$} & {$[1]$} & {$[9]$} & {$[4]$} & This Work \\
\hline Architecture & SAR & SAR & $\Delta \Sigma$ & NS-SAR & NS-SAR \\
\hline Tech. $[\mathrm{nm}]$ & 65 & 65 & 180 & 65 & 90 \\
\hline Vdd $[\mathrm{V}]$ & 0.8 & $0.4-1$ & 1.5 & 1.2 & 1.2 \\
\hline $\mathrm{f}_{\text {Nyq }}[\mathrm{MHz}]$ & 0.08 & 0.02 & 0.20 & 22 & 0.02 \\
\hline Power $[\mu \mathrm{W}]$ & 0.106 & $0.12-0.23$ & 140 & 806 & $0.08-1.5$ \\
\hline ENOB $[\mathrm{bit}]$ & 9.1 & $4.8-8.8$ & 13.7 & 10 & $7.8-11.1$ \\
\hline FoM $[\mathrm{fJ} / \mathrm{cs}]$ & 2.4 & $22-143$ & 105 & 35.8 & $18-53$ \\
\hline
\end{tabular}

energy efficiency of the charge redistribution SAR, while oversampling and noise shaping enable up to $11 \mathrm{~b}$ resolution with an only 8b DAC. The proposed ADC is designed in $90 \mathrm{~nm}$ UMC CMOS and targets acoustic applications with a sampling rate of $320 \mathrm{kS} / \mathrm{s}$ for a signal bandwidth of $10 \mathrm{kHz}$. The dynamic nature of the ADC enables to scale the power from $80.4 \mathrm{nW}$ to $1.49 \mu \mathrm{W}$ as the resolution is varied from 8 to $11 \mathrm{~b}$, to achieve an average FoM of 30fJ/conv-step.

\section{REFERENCES}

[1] M. Yip and A. Chandrakasan, "A Resolution-Reconfigurable 5-to-10-Bit 0.4-to-1V Power Scalable SAR ADC for Sensor Applications," IEEE Journal of Solid-State Circuits, vol. 48, no. 6, pp. 1453-1464, june 2013.

[2] B. Murmann, "ADC Performance Survey 1997-2016," [Online]. Available: http://web.stanford.edu/ murmann/adcsurvey.html, last checked on 2016-11-18.

[3] D. Johns and K. Martin, Analog integrated circuit design: chapter 14 Oversampling converters. New York (N.Y.): Wiley, 1997, iSBN:0-47114448-7.

[4] J.A. Fredenburg and A.M. Flynn, "A 90-MS/s 11-MHz-Bandwidth 62-dB SNDR Noise-Shaping SAR ADC," IEEE Journal of Solid-State Circuits, vol. 47, no. 12, pp. 2898-2904, december 2012.

[5] Razavi, Behzad, "The StrongARM Latch [A Circuit for All Seasons]," IEEE Solid-State Circuits Magazine, vol. 7, no. 2, pp. 12-17, spring 2015.

[6] T. Anderson, "Optimum control logic for successive approximation Analog-to-Digital converters," Comput. Design, vol. 11, no. 7, pp. 81-86, july 1972.

[7] M. Liu, P. Harpe, R. Van Dommele, and A. Van Roermund, "A $0.8 \mathrm{~V}$ 10bit $80 \mathrm{kS} / \mathrm{s}$ SAR ADC with Duty-Cycled Reference Generation," IEEE International Solid-State Circuits Conference, vol. 58, pp. 278-279, march 2015.

[8] P. Harpe, E. Cantatore, and A. Van Roermund, "A 2.2/2.7fJ/conversionstep 10/12b 40kS/s SAR ADC with Data-Driven Noise Reduction," 2013 IEEE International Solid-State Circuits Conference Digest of Technical Papers, pp. 270-271, 2013.

[9] A. Perez, E. Bonizzoni, and F. Maloberti, "A 84dB SNDR 100kHz Bandwidth low-power Single op-amp Third-order $\Delta \Sigma$ Modulator consuming $140 \mu \mathrm{W}, "$ IEEE International Solid-State Circuits Conference, pp. 478480, february 2011. 